\title{
Spatial Vision Deficits in Infants and Children with Down Syndrome
}

\author{
Ffion M. John, ${ }^{1}$ Nathan R. Brombam, ${ }^{1}$ J. Margaret Woodbouse, ${ }^{1}$ and T. Rowan Candy ${ }^{2}$
}

Purpose. Infants and children with Down syndrome show reduced visual acuity and contrast sensitivity when tested with conventional behavioral techniques. These results may reflect sensory deficits of optical or neural origin or a loss of performance in mechanisms responsible for generating the behavioral response. The purpose of this study was to compare objective acuity and contrast sensitivity measurements recorded with visual-evoked potentials (VEPs), with behavioral clinical test results in a group of children with Down syndrome and a group of control subjects. The goal was to determine whether children with Down syndrome still have a sensory deficit when tested using a procedure that is less cognitively demanding than conventional tests.

Methods. The subject group comprised 58 children with Down syndrome and 44 control subjects, aged 3 months to 14.15 years. Visual acuity and contrast sensitivity were measured with steady state, swept VEPs and behavioral techniques. VEP acuity was obtained from 36 children with Down syndrome and 40 control subjects, and behavioral acuity from 54 children with Down syndrome and 35 control subjects. VEP contrast sensitivity was measured in 24 children with Down syndrome and 34 control subjects, and behavioral contrast sensitivity in 42 children with Down syndrome and 25 control subjects. Group differences in visual acuity and contrast sensitivity were analyzed with an analysis of covariance (ANCOVA), with age as a covariate.

Results. Visual acuity thresholds were significantly lower in the group with Down syndrome than in the control group. This was true for both VEP $(P<0.01)$ and behavioral measures $(P<$ $0.01)$. The Down syndrome group also had reduced contrast sensitivity when compared with the control subjects, for VEP contrast sensitivity $(P<0.01)$ and behavioral contrast sensitivity $(P<0.01)$. The group differences remained when children with ophthalmic anomalies were excluded from the analysis.

Conclusions. The reduced visual acuity and contrast sensitivity in the Down syndrome group support the idea of an underlying sensory deficit in the visual system in Down syndrome. (Invest Ophthalmol Vis Sci. 2004;45:1566-1572) DOI:10.1167/ iovs.03-0951

From the ${ }^{1}$ School of Optometry and Vision Sciences, Cardiff University, Cardiff, Wales, United Kingdom; and the ${ }^{2}$ School of Optometry, University of Indiana, Bloomington, Indiana.

Presented in part at the annual meeting of the Association for Research in Vision and Ophthalmology, Fort Lauderdale, Florida, May 2002.

Supported by a National Eye Research Centre grant (FfMJ) and the National Lottery Charities Board in collaboration with Mencap (NRB).

Submitted for publication September 1, 2003; revised December 12, 2003, and January 14, 2004; accepted January 15, 2004.

Disclosure: Ff.M. John, None; N.R. Bromham, None; J.M. Woodhouse, None; T.R. Candy, None

The publication costs of this article were defrayed in part by page charge payment. This article must therefore be marked "advertisement" in accordance with 18 U.S.C. $\$ 1734$ solely to indicate this fact.

Corresponding author: Nathan R. Bromham, School of Optometry and Vision Sciences, Cardiff University, Cardiff CF10 3NB, UK; bromham@cf.ac.uk.
Tnfants and children with Down syndrome show reduced 1 visual acuity and contrast sensitivity when compared with their typically developing peers. ${ }^{1,2}$ These findings may reflect an optical or sensory deficit, since Down syndrome is often associated with ophthalmic anomalies, including refractive errors, ${ }^{3}$ accommodative inaccuracy, ${ }^{4}$ strabismus, ${ }^{5}$ and nystagmus. ${ }^{6}$ Anomalies are also reported in the visual areas of the brain in Down syndrome. These include reduced numbers of neurons and disturbed lamination in the striate cortex, ${ }^{7}$ with altered dendritic and synaptic morphology. ${ }^{8,9}$ Such observations suggest that a neural sensory deficit may also contribute to the reduced visual acuity that occurs in Down syndrome.

Studies of visual acuity and contrast sensitivity in Down syndrome to date have relied on behavioral tests involving motor control, attention and motivation, and sometimes speech and language. Research involving children with Down syndrome have reported reduced arousal, ${ }^{10}$ altered visual attention, ${ }^{11}$ and delayed motor development. It follows that children with Down syndrome may score lower in vision tests, not because they cannot resolve the targets, but because the testing procedure places greater demands on them.

Visual function can be assessed electrophysiologically by recording visual evoked potentials (VEPs) from the scalp over the visual cortex. The use of such tests in children with Down syndrome reduces the requirements for cooperation associated with behavioral testing. Several studies have examined transient VEPs in Down syndrome. Kakigi et al. ${ }^{12}$ reported an increase in the latency and decrease in the amplitude of the P100 component of the pattern-reversal VEP (PRVEP) in adults with Down syndrome. They attributed these differences largely to the common ophthalmic abnormalities of Down syndrome. Fenton et al. ${ }^{13}$ reported a greater latency of the flash P2 component, with normal P100 PRVEP latency in adults with Down syndrome. Increased latency of the flash P2 is a finding often associated with dementia of the Alzheimer's type ${ }^{14}$ and is suggestive of a neural sensory deficit in Down syndrome. The appearance of Alzheimer's disease-like neuropathology in later life is characteristic of Down syndrome. ${ }^{15}$ Ellingson ${ }^{16}$ observed an increased latency of the flash P2 component in newborns with Down syndrome when compared with control subjects. The difference was relatively subtle, and disappeared by 6 months of age. Thus, studies of visual electrophysiology in Down syndrome are suggestive of anomaly, but it is not clear how these findings might relate to visual function.

In the present study, we sought to compare objective acuity and contrast sensitivity measurements recorded by steady state pattern-reversal VEPs with behavioral clinical test results in a group of children with Down syndrome and a group of agematched control children. Whereas behavioral vision tests also involve the child's higher visual, motor, and central processing, ${ }^{17}$ the VEP reflects the integrity of the visual pathways up to the level of the primary visual cortex. ${ }^{18} \mathrm{VEP}$ tests are less demanding from the child's perspective, requiring only stable fixation, and the simplicity of the test should reduce the influence of cognitive and motivational factors on visual thresholds in children with Down syndrome. 


\section{Methods}

Since 1991, the visual development of a large group of children with Down syndrome has been studied at the School of Optometry and Vision Sciences of Cardiff University. Fifty-eight children from this group, 35 boys and 23 girls, were recruited into the present study. The children with Down syndrome were aged between 9 months and 12.75 years, with a mean age of 6.66 years. All the children with Down syndrome had full trisomy 21, confirmed by cytogenetic testing. A further 44 typically developing children, 30 boys and 14 girls, were recruited as a control group. The control group was aged between 3 months and 14.15 years, with a mean age of 4.55 years. These children were either siblings of the children with Down syndrome or were recruited through contacts within the Department of Optometry and Vision Sciences. The recruitment and experimental protocols for the study were conducted in compliance with the Declaration of Helsinki and approved by local ethical research committees.

Refractive error was measured using Mohindra retinoscopy. ${ }^{19}$ The group with Down syndrome contained 7 myopes $(<-0.5 \mathrm{D}), 33$ hyperopes $(>+2.0 \mathrm{D})$, and 19 emmetropes. The control group contained 5 myopes, 1 hypermetrope, and 38 emmetropes. Ametropes wore their habitual correction during the visual acuity and contrast sensitivity testing. The children with Down syndrome visit the School of Optometry on a regular basis, allowing spectacle prescriptions to be checked. Accommodation was assessed with modified Nott dynamic retinoscopy ${ }^{4}$ at target distances of $10,6.25$, and $4 \mathrm{D}$. Accommodation was considered accurate if the measured response was within $0.75 \mathrm{D}$ of the target for at least two of the target distances. Eye alignment was assessed using the Hirshberg test and a near cover test. Nystagmus was detected by direct observation of the children's eye movements while fixating targets. Nystagmus in Down syndrome is typically fine and horizontal $^{6}$ and all the children underwent ophthalmoscopy which would have allowed detection of very fine amplitude nystagmus not visible by observation but no additional cases were found. Binocular visual acuity and contrast sensitivity were measured using the steady state swept VEP (software provided by Anthony M. Norcia ${ }^{20}$ ) and ability-appropriate behavioral techniques.

\section{Steady State VEP Procedure}

The active electrodes were placed at $\mathrm{O}_{\mathrm{Z}}, \mathrm{O}_{1}$, and $\mathrm{O}_{2}$ and referenced to $\mathrm{C}_{\mathrm{Z}}$. The ground electrode was placed at $\mathrm{P}_{\mathrm{Z}}$. Gold-cup surface electrodes (E-6H; Grass Telefactor Corp., W. Warwick, RI) were used. The electroencephalogram (EEG) signal was digitized over a $0.1-$ to $100-\mathrm{Hz}$ bandwidth, at a sampling rate of $600 \mathrm{~Hz}$. Preamplifier gain was set to 20,000 for young infants and 50,000 for the other subjects.

The stimulus for the VEP grating acuity protocol was a vertical sine wave of $80 \%$ fixed contrast that was undergoing counterphase reversal at $5 \mathrm{~Hz}$. The space average luminance of the grating patch was 85 $\mathrm{cd} / \mathrm{m}^{-2}$. During each trial, the spatial frequency of this patch increased linearly once every second for 11 seconds. The range of frequencies tested was selected on the basis of the child's behavioral acuity threshold. In cases in which a child's behavioral threshold was unknown, the spatial frequency range was estimated from the behavioral thresholds of a child of similar age. The upper limit of the spatial frequency range was limited by the display resolution and the viewing distance, and therefore, to allow for greater sweep ranges, older children had to be tested at greater viewing distances. The size of the grating patch was $20.00,14.07$, and $9.41 \mathrm{deg}^{2}$ at 70-, 100-, and 150-cm viewing distances, respectively. Sweep ranges were 1.30 to $13.72,2.00$ to 19.60 and 1.76 to $29.40 \mathrm{cyc} / \mathrm{deg}$ for the viewing distances of 70,100 , and $150 \mathrm{~cm}$, respectively. The step sizes between each 1 -second recording bin were $1.24,1.76$, and $2.64 \mathrm{cyc} / \mathrm{deg}$ for $70-, 100-$, and $150-\mathrm{cm}$ viewing distances, respectively.

The stimulus for the contrast sensitivity recording was a vertical 2 cyc/deg sine wave, also at a temporal frequency of $5 \mathrm{~Hz}$. The size and average luminance of the patch were the same as for the VEP acuity test. The contrast of the grating decreased logarithmically once every second during the 11-second trial. The range of the contrast values used was either $30 \%$ to $0.3 \%$, or $20 \%$ to $0.2 \%$ contrast, depending on behavioral thresholds.

Subjects completed six, 11-second trials for both contrast and acuity stimuli. A small fixation toy, dangled in front of the screen, helped to elicit fixation and maximize the children's accommodative accuracy (Candy T, et al. IOVS 2002; 43:ARVO E-Abstract 2865). There were several toys that were interchanged to maintain the child's interest. An observer monitored the child, pausing the EEG recording if the child looked away or moved excessively.

The technique used to score the data was based on that of Norcia and Tyler. ${ }^{20}$ An experienced observer scored the data, unaware of the age and subject group (Down syndrome versus control) of each child. Scoring involved the use of a series of discrete Fourier transforms that provided the amplitude and phase of the evoked response in 1-second bins over the trial. The VEP response amplitude at the second harmonic $(10 \mathrm{~Hz})$ of the stimulus temporal frequency was used to estimate the stimulus-driven component of the EEG. The baseline EEG response amplitudes at 8 and $12 \mathrm{~Hz}$ were used as a noise estimate for comparison to the $10 \mathrm{~Hz}$ visually driven signal. Acuity thresholds were estimated by linear extrapolation to 0 amplitude of the function relating VEP amplitude $(10 \mathrm{~Hz})$ to stimulus spatial frequency. Contrast thresholds were similarly estimated by extrapolation to 0 of the function relating VEP amplitude $(10 \mathrm{~Hz})$ to $\log$ stimulus contrast. The criteria for the range used for the extrapolation were: The function had to be monotonically decreasing within the range, the signal in the first bin in the range had to exceed the noise by a factor of 3 , and the phase of the signal had to be consistent. When the VEP data contained more than one region that met the scoring criteria, the region yielding the best threshold was scored. For both visual acuity and contrast sensitivity, the final threshold was taken as the best of the thresholds recorded on the three channels.

\section{Behavioral Vision Tests}

Behavioral visual acuity and contrast sensitivity were measured using tests appropriate to each child's ability. Because visual thresholds are influenced by the test used, a more valid comparison would result if the same behavioral acuity test were used for all subjects. This was not possible, because young infants are only testable with preferentiallooking techniques, but such techniques are inappropriate for older children, either because the repetitive nature of the test becomes tedious or because the test ceiling is reached. To establish the behavioral threshold, it was important that the end point of the test not be reached. We therefore chose to vary the test, depending on the child's age and ability, as would be done in a clinical situation.

The tests used for visual acuity, in order of age and ability, consisted of Teller Acuity Cards, Cardiff Acuity Test (both preferential-looking tests), Kay Picture Test, logMAR Crowded Test (logMAR picture and letter optotype tests used as matching or naming tests), and the Bailey-Lovie letter chart. The Cardiff Contrast Test and Pelli Robson Contrast Sensitivity Chart were used to test contrast sensitivity. It is known that accommodative inaccuracy is common in children with Down syndrome, ${ }^{21}$ and therefore, wherever possible, the behavioral tests were conducted at the same viewing distance as the VEP.

\section{Results}

The number of children tested in each paradigm, age range, and subject group is shown in Table 1. Twelve (21\%) of the 58 children with Down syndrome and $4(9 \%)$ of the 44 control subjects failed to produce a criterion VEP response to the acuity stimulus. VEP acuity therefore could not be estimated in these children. A further 10 (17\%) of the children with Down syndrome where not sufficiently compliant to complete the VEP acuity testing procedure. VEP acuity was obtained from 36 (62\%) of the children with Down syndrome and from 40 (91\%) of the control subjects. Behavioral acuity was successfully 
Table 1. Number of Children Tested, by Paradigm, Age Range and Subject Group

\begin{tabular}{|c|c|c|c|c|c|c|c|c|c|}
\hline \multirow[b]{2}{*}{$\begin{array}{c}\text { Age Range } \\
\text { (y) }\end{array}$} & \multirow[b]{2}{*}{$n$} & \multicolumn{4}{|c|}{ Visual Acuity } & \multicolumn{4}{|c|}{ Contrast Sensitivity } \\
\hline & & $\begin{array}{c}\text { Behavioral } \\
\text { Thresholds } \\
\text { Obtained } \\
\text { (n) }\end{array}$ & $\begin{array}{c}\text { VEP } \\
\text { Thresholds } \\
\text { Obtained } \\
\text { (n) }\end{array}$ & $\begin{array}{c}\text { Insignificant } \\
\text { VEP } \\
\text { Responses } \\
\text { (n) }\end{array}$ & $\begin{array}{c}\text { VEP } \\
\text { Noncompliance } \\
(n)\end{array}$ & $\begin{array}{c}\text { Behavioral } \\
\text { Thresholds } \\
\text { Obtained } \\
\text { (n) }\end{array}$ & $\begin{array}{c}\text { VEP } \\
\text { Thresholds } \\
\text { Obtained } \\
\text { (n) }\end{array}$ & $\begin{array}{c}\text { Insignificant } \\
\text { VEP } \\
\text { Responses } \\
\text { (n) }\end{array}$ & $\begin{array}{c}\text { VEP } \\
\text { Noncompliance } \\
(n)\end{array}$ \\
\hline \multicolumn{10}{|l|}{ DS } \\
\hline $0-2$ & 5 & 5 & 5 & - & - & 2 & 2 & 2 & 1 \\
\hline $2-4$ & 6 & 6 & 2 & 2 & 2 & 4 & 1 & 3 & 2 \\
\hline $4-6$ & 12 & 11 & 7 & 4 & 1 & 9 & 7 & 3 & 2 \\
\hline $6-8$ & 16 & 13 & 7 & 2 & 7 & 14 & 5 & 4 & 7 \\
\hline $8-10$ & 11 & 11 & 8 & 3 & - & 9 & 4 & 6 & 1 \\
\hline $10-14$ & 8 & 8 & 7 & 1 & - & 4 & 5 & 3 & - \\
\hline Total & 58 & 54 & 36 & 12 & 10 & 42 & 24 & 21 & 13 \\
\hline \multicolumn{10}{|l|}{ Control } \\
\hline $0-2$ & 16 & 10 & 15 & 1 & - & 5 & 10 & 3 & 1 \\
\hline $2-4$ & 8 & 7 & 7 & 1 & - & 6 & 5 & 4 & 1 \\
\hline $4-6$ & 7 & 7 & 7 & - & - & 5 & 6 & 1 & - \\
\hline $6-8$ & 3 & 3 & 2 & 1 & - & 3 & 3 & - & - \\
\hline $8-10$ & 5 & 4 & 5 & - & - & 3 & 5 & - & - \\
\hline $10-14$ & 5 & 4 & 4 & 1 & - & 3 & 5 & - & - \\
\hline Total & 44 & 35 & 40 & 4 & 0 & 25 & 34 & 8 & 2 \\
\hline
\end{tabular}

Insignificant VEP responders were those infants and children who failed to produce a criterion VEP response. Thresholds could not be estimated in this group. The VEP noncompliance category includes those who refused to allow electrodes to be put on and those cases in which the VEP test had to be aborted.

measured in $54(93 \%)$ of the children with Down syndrome and in 35 (80\%) of the control subjects. Criterion VEP responses to the contrast sensitivity stimulus were not elicited in $21(36 \%)$ of the children with Down syndrome and in 8 (18\%) of the control subjects. Thirteen (22\%) of the children with Down syndrome and 2 (5\%) of the control subjects where not sufficiently compliant to complete the VEP contrast sensitivity test. VEP contrast sensitivity was obtained from 24 (41\%) of the children with Down syndrome and $34(77 \%)$ of the control subjects and behavioral contrast sensitivity from $42(74 \%)$ of the children with Down syndrome and 25 (57\%) of the control subjects.

\section{Visual Acuity}

Behavioral visual acuity data are shown in Figure 1A and VEP visual acuity data in Figure $1 \mathrm{~B}$. The visual acuity data are summarized in Figure 1C by dividing the children into the age
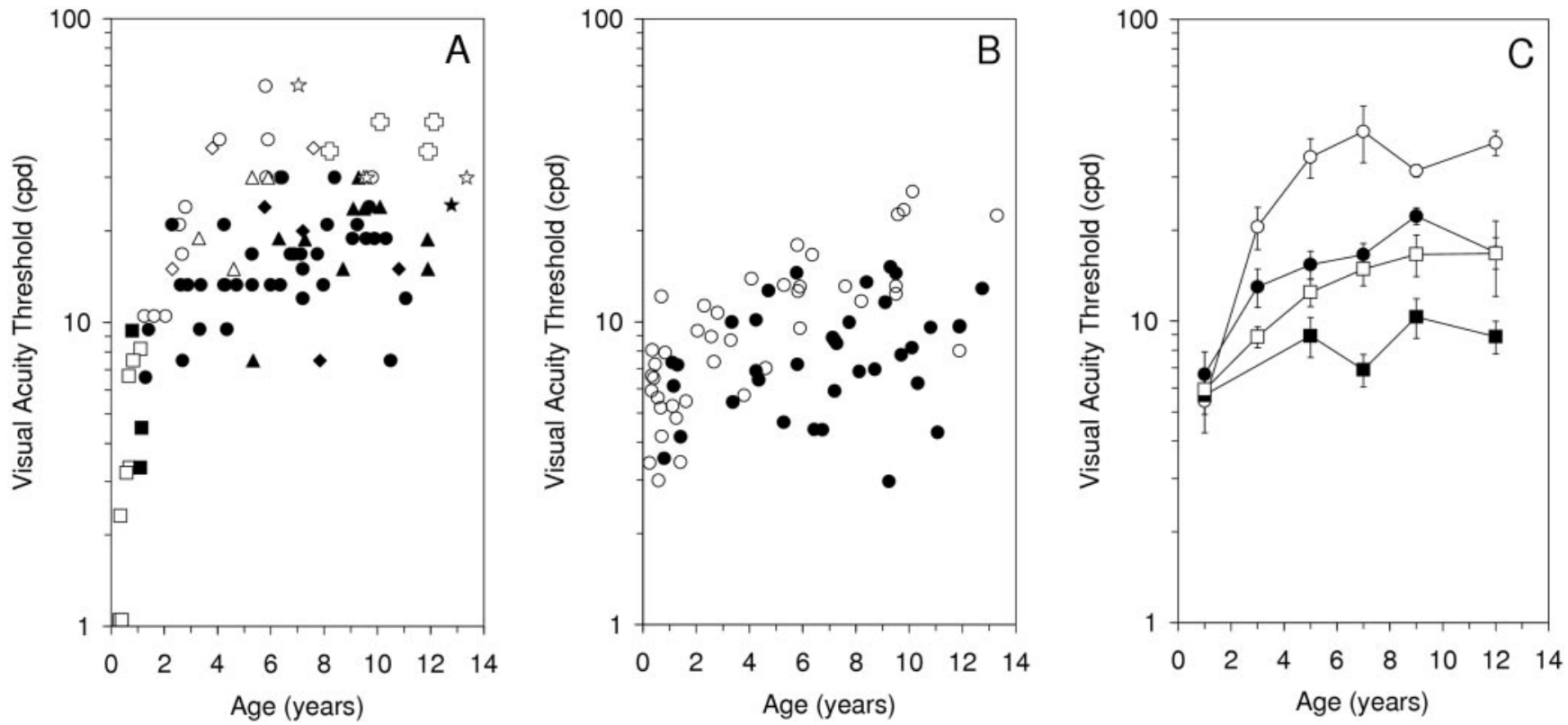

Figure 1. (A) Behavioral visual acuity threshold (in cycles per degree) for Down syndrome (filled symbols) and control (open symbols) subjects versus age in years. The behavioral test used in each case is shown as follows: Teller Acuity Cards, squares; Cardiff Acuity Cards, circles; Kay Picture Test, diamonds; LogMAR Crowded Test, triangles; Snellen Chart, pluses; and Bailey-Lovie letter chart, stars. (B) VEP visual acuity threshold (in cycles per degree) for Down syndrome (filled symbols) and control (open symbols) versus age in years. (C) The behavioral (circles) and VEP (squares) acuity data for Down syndrome (filled symbols) and control (open symbols) groups, summarized by taking averages within the age groups shown in Table 1 . Error bars = SEM. Data from age groups with fewer than three children are not included. 
Figure 2. (A) VEP versus behavioral VA (in cycles per degree), for Down syndrome (filled symbols) and control (open symbols) children. (B) The ratio of VEP to behavioral visual acuity (octaves) versus age in years. When VEP is greater than behavioral VA, this ratio is positive; and, when behavioral VA is the greater, the ratio is negative.
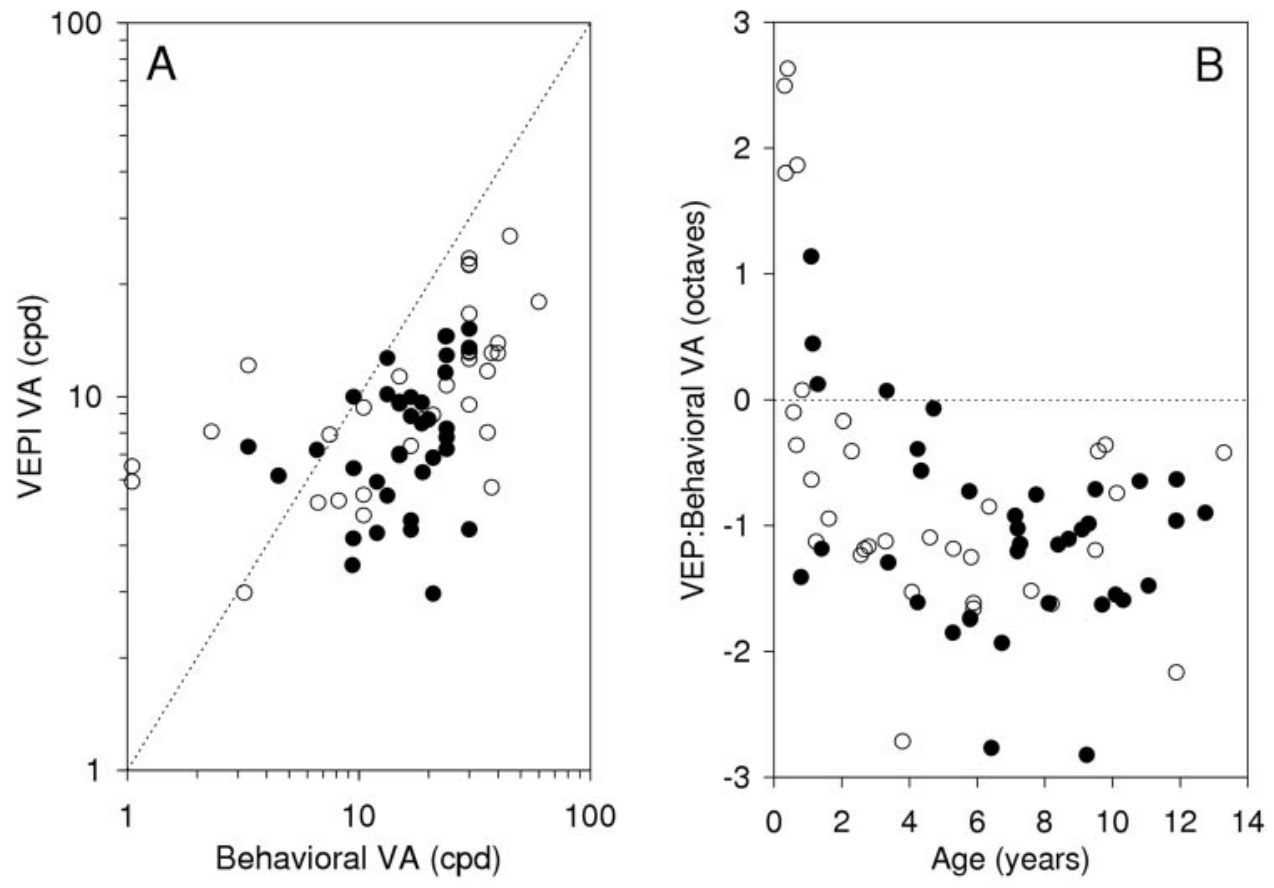

groups shown in Table 1 and taking the mean result. The differences between VEP and behavioral acuity are shown more clearly in Figure 2.

A one-way between-groups analysis of covariance (ANCOVA) was used to compare VEP visual acuity in the Down syndrome and control groups. The independent variable was the subject group (Down syndrome, control) and the dependent variable was the VEP visual acuity threshold. Subject age was used as the covariate, because visual acuity is known to improve with age in both groups. ${ }^{1}$

There was a significant difference between the VEP visual acuity of the two groups $\left(\mathrm{F}_{(1,73)}=20.15, P<0.01, \eta^{2}=0.22\right)$. Using similar ANCOVA, a significant difference between the behavioral visual acuity of the groups was also observed $\left(\mathrm{F}_{(1,86)}\right.$ $\left.=35.31, P<0.01, \eta^{2}=0.29\right)$. The group with Down syndrome had significantly lower visual acuity for both VEP and behavioral measures.

The vertically oriented grating pattern-reversal stimuli used in this study were not optimal for children with nystagmus, ${ }^{22}$ which usually has a horizontal component. Strabismus limits binocular summation and reduces binocular visual acuity. Inaccurate accommodation in the children with Down syndrome could contribute to reduced visual acuity. For this reason, 35 children from the Down syndrome group were excluded because they had any combination of inaccurate accommodation (28 cases), nystagmus ( 9 cases), or strabismus ( 9 cases). A further seven children with Down syndrome and nine control subjects who had not had their accommodation measured were also excluded. This left 16 children with Down syndrome and 35 control subjects. ANCOVA was used to analyze the remaining data. The significant group differences remained, for VEP acuity $\left(\mathrm{F}_{(1,47)}=16.97, P<0.01, \eta^{2}=0.27\right)$ and behavioral acuity $\left(\mathrm{F}_{(1,45)}=13.30, P<0.01, \eta^{2}=0.23\right)$.

The VEP and behavioral acuity measures were compared for the 36 children with Down syndrome and 33 control subjects who had completed both tests. All children were included regardless of ocular anomalies. There was a positive correlation between VEP and behavioral VA in both the Down syndrome $(r=0.42, P=0.01)$ and control $(r=0.63, P<0.01$; Fig. $2 \mathrm{~A})$ groups. The ratio of VEP to behavioral acuity in octaves was calculated (Fig. 2B). Behavioral VA was better than VEP VA in 32 (89\%) of the 36 children with Down syndrome and in 28 (85\%) of the 33 control subjects. Cases in which VEP VA was superior were found only in the youngest age groups. Paired samples $t$-tests were conducted to compare VEP and behavioral acuity in the two subject groups. All children were included regardless of ocular anomalies. For the Down syndrome group, there was a statistically significant difference between behavioral acuity (mean, $17.37 \pm 6.94 \mathrm{cyc} / \mathrm{deg}[\mathrm{SD}]$ ) and VEP acuity (mean, $8.23 \pm 3.35 \mathrm{cyc} / \mathrm{deg}$ ): $\left.t_{(35)}=8.68, P<0.01\right)$. A difference was also found between control group behavioral acuity (mean, $22.65 \pm 14.93 \mathrm{cyc} / \mathrm{deg}$ ) and VEP acuity (mean, $11.34 \pm 5.95 \mathrm{cyc} / \mathrm{deg}): t_{(32)}=5.39, P<0.01$

\section{Contrast Sensitivity}

Behavioral contrast sensitivity data are shown in Figure 3A and VEP contrast sensitivity data are shown in Figure 3B. The contrast sensitivity data are summarized in Figure $3 \mathrm{C}$ by dividing the children into the age groups shown in Table 1 .

A one-way, between-groups ANCOVA was used to compare contrast sensitivity in the Down syndrome and control groups, with subject age as the covariate. A $\log$ transformation was applied to the original contrast sensitivity values, because the data were not normally distributed. Significant differences between the two groups were observed, with the Down syndrome group having lower contrast sensitivity for both measures. For VEP contrast sensitivity $\left(\mathrm{F}_{(1,55)}=15.33, P<0.01, \eta^{2}\right.$ $=0.22)$ and behavioral contrast sensitivity $\left(\mathrm{F}_{(1,64)}=10.54, P<\right.$ $\left.0.01, \eta^{2}=0.14\right)$.

After the children with ocular anomalies were excluded from the analysis, there was still a difference between the two groups for behavioral contrast sensitivity $\left(\mathrm{F}_{(1,29)}=10.25, P<\right.$ $\left.0.01, \eta^{2}=0.26\right)$ and VEP contrast sensitivity $\left(\mathrm{F}_{(1,28)}=7.465\right.$, $P=0.01, \eta^{2}=0.21$ )

Paired samples $t$-tests were used to compare the log-transformed VEP and behavioral contrast sensitivities of the 23 children with Down syndrome and 21 control subjects who had completed both tests. Children were included regardless of ocular anomalies. In the Down syndrome group, there was no significant difference between log behavioral contrast sen- 

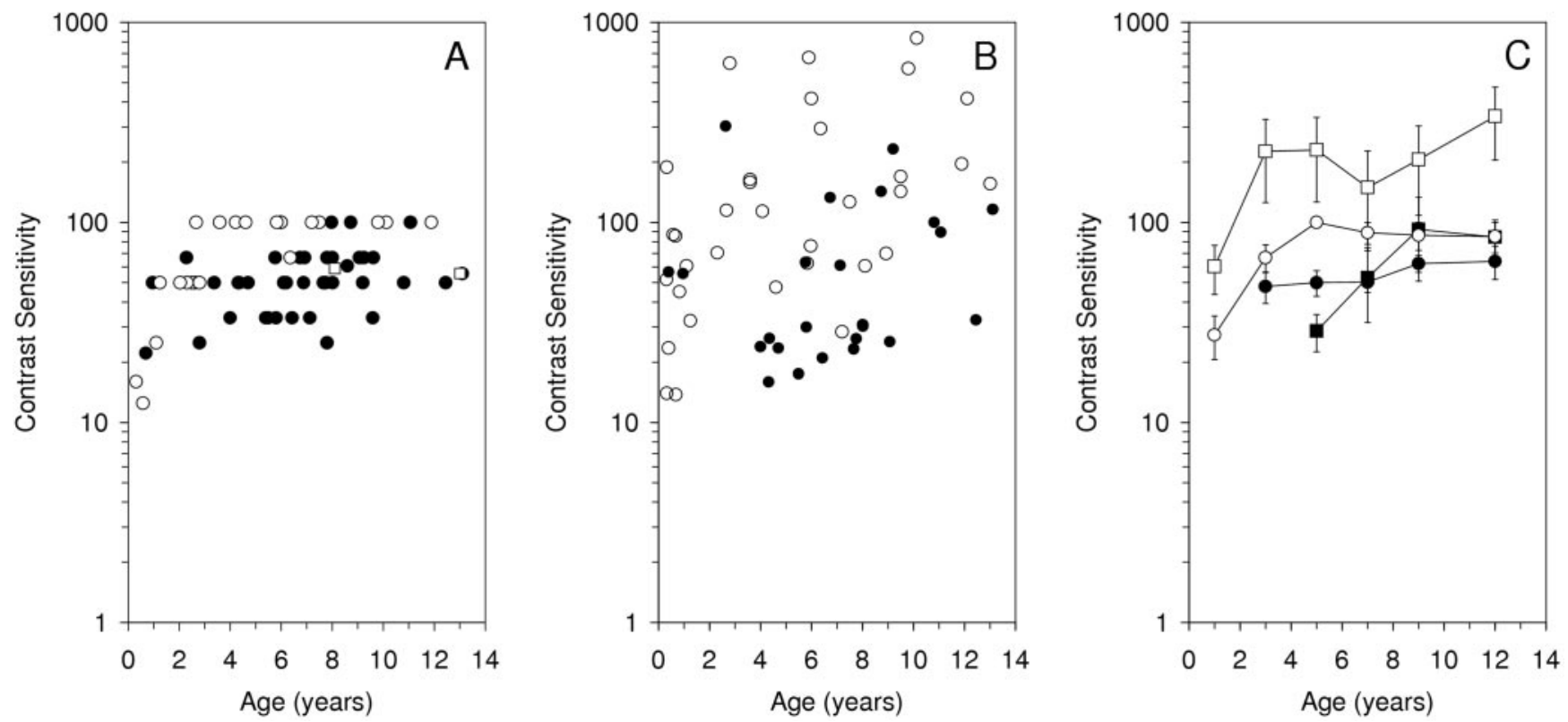

FiguRe 3. (A) Behavioral contrast sensitivity for Down syndrome (filled symbols) and control (open symbols) versus age in years. The behavioral test used in each case is shown as follows: Cardiff Contrast Test, circles; Pelli Robson Contrast Sensitivity Chart, squares. (B) VEP contrast sensitivity for Down syndrome (filled symbols) and control (open symbols) versus age in years. (C) Behavioral (circles) and VEP (squares) contrast sensitivities for Down syndrome (filled symbols) and control (open symbols) groups, summarized by taking averages within the age groups shown in Table 1. Error bars $=$ SEM. Age groups with fewer than three children are not included.

sitivity (mean, $1.71 \pm 0.13[\mathrm{SD}]$ ) and $\log$ VEP contrast sensitivity (mean, $1.68 \pm 0.37$ ): $t_{(22)}=0.48, P=0.64$. There was a statistically significant difference between control group log behavioral contrast sensitivity (mean, $1.82 \pm 0.28$ ) and log VEP contrast sensitivity (mean, $2.08 \pm 0.43$ ): $t_{(21)}=-2.70, P<$ 0.01 .

\section{Discussion}

The children with Down syndrome had reduced visual acuity and contrast sensitivity when compared with typically developing children. This was true for both VEP and behavioral tests. Our results agree with previous studies of vision in Down syndrome which used behavioral tests alone $e^{1,2,23}$ and are consistent with the notion of a primary sensory deficit in Down syndrome.

Norcia and Tyler, ${ }^{20}$ using the steady state VEP technique, showed that visual acuity develops rapidly over the earliest months of life and then begins to slow down at the end of the first year after reaching around $20 \mathrm{cyc} / \mathrm{deg}$. Few infants were tested in the present study, and so this early phase of development could not be observed, but considerable improvement in visual acuity occurred over the first 2 years of life. Average VEP acuities did not reach the $20 \mathrm{cyc} / \mathrm{deg}$ reported at 1 year by Norcia and Tyler $^{20}$ even in the 10- to 14-year-old control subjects. Visual thresholds obtained with VEPs are highly dependent on stimulus conditions such as luminance, ${ }^{24}$ field size ${ }^{25}$ and temporal frequency. In the present study, we used a lower display luminance and grating temporal frequency than did Norcia and Tyler. ${ }^{20}$ Behavioral visual acuity thresholds in the control group were in general agreement with previous studies, showing rapid initial development followed by a more gradual improvement thereafter. ${ }^{26}$

It could be argued that the reduced VEP thresholds in the younger children and in the Down syndrome group are explained, at least in part, by poorer fixation, visual attention, and accommodation. We attempted to optimize visual fixation by carefully monitoring gaze during the VEP recording and by changing the fixation targets regularly to maintain the interest of the children. Reduced VEP acuity in the Down syndrome group was still seen when children with poor accommodation were excluded from the analysis, (although we cannot say for certain that the remaining children were accommodating accurately, even though they had the facility). There is evidence that the amplitude of the steady state VEP can be modified by attention, independent of visual fixation. ${ }^{27,28}$ Di Russo et al. ${ }^{27}$ observed that when subjects actively attend a stimulus, rather than just fixate its spatial position, the amplitude of the associated steady state VEP response is increased and its latency decreased. They suggest the visual system may prioritize visualevoked signals on the basis of their amplitude and latency, modulating the signals through cortical contrast gain control mechanisms. Many investigators have argued that visual attention is anomalous in Down syndrome because children with the syndrome tend to exhibit longer visual fixations. ${ }^{11,29}$ It follows that, even when children fixate and accommodate accurately, if they do not actively attend the stimulus, VEP thresholds will be reduced.

Infant visual acuity studies using steady state VEP and behavioral Forced-Choice Preferential Looking (FPL) measures of acuity generally find that VEP acuity is better than behavioral. ${ }^{24,30}$ Katsumi et al. ${ }^{31}$ found that, in adults, behavioral acuity is between 0.5 and 1 octave greater than steady state VEP acuity. In agreement with Katsumi et al., behavioral acuity in the older children in our study was approximately 1 octave better than acuity measured using the VEP. It appears that, at some point after infancy, behavioral becomes superior to VEP visual acuity, and in the present study this point was between 1 and 2 years of age, in both the Down syndrome and control groups. It is possible that this point simply reflects the use of a different behavioral acuity test, because it coincided with the change from the Teller Cards to the Cardiff Acuity Test. It is perhaps not surprising that different developmental trajectories are observed for VEP and behavioral measures of vision, 
because they tap different mechanisms. ${ }^{30}$ Important stimulus and procedural differences between the behavioral and VEP tests used in the present study should also be noted when making direct comparisons between the measures. For example, the VEP stimulus was temporally modulated, whereas the behavioral one was not, and there were differences between the field size, scoring criteria, and luminance of the VEP and behavioral tests.

The development of VEP contrast sensitivity to gratings of 2 cyc/deg is relatively rapid, with sensitivity approaching adult levels before 6 months of age. ${ }^{32,33}$ In the present study, little change occurred in VEP contrast sensitivity beyond the first year, and the contrast sensitivities of the control group were on the order of those reported by Norcia et al. ${ }^{32}$ Developmental studies of contrast sensitivity using behavioral measures suggest that sensitivity is adultlike at approximately 8 years. ${ }^{34,35}$ Behavioral contrast sensitivities in the present study showed development over the first 2 years, but it is likely that further improvement was masked as children reached the ceiling of the Cardiff contrast test. The results of the acuity tests predict that behavioral contrast sensitivity should be superior to VEP contrast sensitivity. This was not the case, at least for the control children, probably because of the ceiling effect of the Cardiff contrast test. Problems were experienced in generating criterion VEP responses to the contrast stimulus in the Down syndrome group. Crow et al. ${ }^{36}$ also report difficulties when using a similar VEP contrast sensitivity paradigm in elderly adults with Alzheimer's disease. They suggest that age-related decreases in the amplitude and increases in the latency of the flash and pattern VEPs may relate to their difficulty in recording consistent VEP responses. It is possible that such factors were at work in the present study, since signs of Alzheimer's neuropathology have been noted in persons as young as 8 years of age in Down syndrome. ${ }^{37}$

Ophthalmic anomalies cannot entirely account for reduced vision in Down syndrome, because the children without such anomalies in this study still showed poorer visual acuity and contrast sensitivity. Courchesne ${ }^{38}$ suggested that abnormalities in cortical sensory evoked potentials in Down syndrome reflect the characteristic neural abnormalities in the Down syndrome cortex and cerebellum. Studies of the brain in Down syndrome suggest both developmental failure and processes of neurodegeneration later in life. ${ }^{15}$ Both congenital abnormality and age-related neuropathology within the visual pathways in Down syndrome could account for the reduced spatial vision in this study. The involvement of neural anomalies at earlier stages of the visual pathways cannot be ruled out, because there are few published data on the structure and function of the retina in Down syndrome.

The implications of this study are that, even when children with Down syndrome are free from ophthalmic anomalies, they experience sensory visual impairment that cannot be fully corrected with spectacles.

\section{References}

1. Woodhouse JM, Pakeman VH, Saunders KJ, et al. Visual acuity and accommodation in infants and young children with Down's syndrome. J Intellect Disabil Res. 1996;40:49-55.

2. Courage ML, Adams RJ, Reyno S, Kwa PG. Visual acuity in infants and children with Down syndrome. Dev Med Child Neurol. 1994; 36:586-593.

3. Woodhouse JM, Pakeman VH, Cregg M, et al. Refractive errors in young children with Down syndrome. Optom Vis Sci. 1997;74: 844-851.

4. Cregg M, Woodhouse JM, Pakeman VH, et al. Accommodation and refractive error in children with Down syndrome: cross-sectional and longitudinal studies. Invest Ophthalmol Vis Sci. 2001;42:5563.

5. Haugen $\mathrm{OH}$, Hodvig G. Strabismus and binocular function in children with Down syndrome: a population-based, longitudinal study. Acta Opbthalmol Scand. 2001;79:133-139.

6. Wagner RS, Caputo AR, Reynolds RD. Nystagmus in Down's Syndrome. Ophthalmology. 1990;97:1439-1443.

7. Wisniewski KE, Kida E, Brown WT. Consequences of genetic abnormalities in Down's Syndrome on brain structure and function. In: Rondal JA, Perera J, et al., eds. Down's Syndrome. Psychological, Psychobiological and Socio-Educational Perspectives. London: Whurr; 1996:3-19.

8. Becker L, Mito T, Takashima S, Onodera K. Growth and development of the brain in Down syndrome. In: The Morphogenesis of Down Syndrome. New York: Wiley-Liss; 1991;133-152.

9. Ida K, Tachashima S, Mito T, Yao R, Onodera K. Immuno-histochemical and Golgi studies on brain development and aging in patients with Down syndrome. Ital J Intellect Impair. 1993;6:311.

10. Wagner S, Ganiban JM, Cicchetti D. Attention, memory and perception in infants with Down syndrome: a review and commentary. In: Cicchetti D, Beeghly M, eds. Children with Down Syndrome, A Developmental Perspective. Cambridge, UK: Cambridge University Press; 1990;147-179.

11. Miranda SB, Fantz RI. Visual preferences of Down's syndrome and normal Infants. Child Dev. 1973;44:555-561.

12. Kakigi R, Oono S, Matsuda Y, Kuroda Y. Pattern-reversal visual evoked potentials in Down's Syndrome. Acta Neurol Scand. 1993; $87: 410-415$.

13. Fenton G, Jones A, Forbes A. Evoked potentials in Down's syndrome. Electroencephalogr Clin Neurophysiol. 1996;99:354.

14. Moore N, Tucker K, Jann M, Hostetler R, Coburn K. Flash P2 delay in primary degenerative dementia of the Alzheimer type. Prog Neuropsychopharmacol Biol Psychiatry. 1995;19:403-410.

15. Engidawork E, Lubec G. Molecular changes in fetal Down syndrome brain. J Neurochem. 2003;84:895-904.

16. Ellingson R. Development of visual evoked potentials and photic driving responses in normal full term, low risk premature, and trisomy-21 infants during the first year of life. Electroencephalogr Clin Neuropbysiol. 1986;63:309-316.

17. Teller DY. First glances: the vision of infants. The Friedenwald Lecture. Invest Opbthalmol Vis Sci. 1997;38:2183-2203.

18. Regan D. Human Brain Electropbysiology. New York: Elsevier Press; 1989.

19. Mohindra I. A technique for infant examination. Am J Optom Pbysiol Optics. 1975;54:319-322.

20. Norcia AM, Tyler CW. Spatial frequency sweep VEP: visual acuity during the first year of life. Vis Res. 1985;25:1399-1408.

21. Woodhouse JM, Meades JS, Leat SJ, Saunders KJ. Reduced accommodation in children with Down syndrome. Invest Ophthalmol Vis Sci. 1993;34:2382-2387.

22. Saunders KJ, Brown G, McCulloch DL. Pattern-onset visual evoked potentials: more useful than reversal for patients with nystagmus. Doc Opbthalmol. 1998;94:265-274.

23. Courage ML, Adams RJ, Hall EJ. Contrast sensitivity in infants and children with Down syndrome. Vis Res. 1997;37:1545-1555.

24. Allen D, Bennet P, Banks MS. The effects of luminance on FPL and VEP acuity in human infants. Vis Res. 1992;32:2005-2012.

25. Campbell FW, Maffei L. Electrophysiological evidence for the existence of orientation and size detectors in the human visual system. J Physiol. 1970;207:635-652.

26. Salomao S, Ventura D. Large sample population age norms for visual acuities obtained with Vistech-Teller acuity cards. Invest Ophthalmol Vis Sci. 1995;36:657-670.

27. Di Russo F, Spinelli D, Morrone MC. Automatic gain control contrast mechanisms are modulated by attention in humans: evidence from visual evoked potentials. Vis Res. 2001;41:2435-2447.

28. Di Russo F, Spinelli D. Effects of sustained, voluntary attention on amplitude and latency of steady-state visual evoked potential: a costs and benefits analysis. Neurophysiol Clin. 2002;113:17711777. 
29. Cohen LB. Examination of habituation as a measure of aberrant infant development. In: Friedman SL, Sigman M, eds. Preterm Birth and Psychological Development. New York: Academic Press; 1981;241-253.

30. Banks MS, Dannemiller JL. Infant visual psychophysics. In: Salapatek P, Cohen L, eds. Handbook of Infant Perception. New York: Academic Press; 1987;115-184.

31. Katsumi O, Arai M, Wajima R, Denno S, Hirose T. Spatial frequency sweep pattern reversal VER acuity vs Snellen visual acuity: effect of optical defocus. Vis Res. 1995;36:903-909.

32. Norcia AM, Tyler CW, Hamer RD. Development of contrast sensitivity in the human infant. Vis Res. 1990;30:1475-1486.

33. Allen D, Tyler CW, Norcia AM. Development of grating acuity and contrast sensitivity in the central and peripheral field of the human infant. Vis Res. 1995;36:1945-1953.
34. Ellemberg D, Lewis TL, Liu C, Maurer D. Development of spatial and temporal vision during childhood. Vis Res. 1999;39:23252333.

35. Adams RJ, Courage ML. Using a single test to measure human contrast sensitivity from early childhood to maturity. Vis Res. 2002; 42:1205-1210.

36. Crow RW, Levin LB, LaBree L, Rubin R, Feldon SE. Sweep visual evoked potential evaluation of contrast sensitivity in Alzheimer's dementia. Invest Ophthalmol Vis Sci. 2003;44:875-878.

37. Leverenz JB, Raskind MA. Early amyloid deposition in the medial temporal lobe of young Down syndrome patients. Exp Neurol. 1998; 150:296-304.

38. Courchesne E. Physioanatomical considerations in Down syndrome. In: Nadel L, ed. The Psychobiology of Down Syndrome. Cambridge, MA: MIT Press; 1988;291-313. 